\title{
On Fuzzy Differential Subordination
}

\begin{abstract}
A. HAYDAR EŞ
Abstract. The theory of differential subordination was introduced by S.S.Miller and P.T.Mocanu in [2], then developed in many papers. In [1] the authors investigate various subordination results for some subclasses of analytic functions in the unit disc. G.I.Oros and G.Oros define the notion of fuzzy subordination and in $[3,4,5]$ they define the notion of fuzzy differential subordination. In this paper, we determine sufficient conditions for a multivalent function to be a dominant of the fuzzy differential subordination.
\end{abstract}

\section{INTRODUCTION}

We introduce some basic notions and results that are used in the sequel.

Definition 1.1 ([6]). Let $X$ be a non-empty set. An application $F: X \rightarrow$ $[0,1]$ is called fuzzy subset. An alternate definition, more precise, would be the following: A pair $\left(A, F_{A}\right)$, where $F_{A}: X \rightarrow[0,1]$ and

$$
A=\left\{x \in X: 0<F_{A}(x) \leq 1\right\}=\operatorname{supp}\left(A, F_{A}\right),
$$

is called fuzzy subset.

Proposition $1.1([3])$. If $\left(M, F_{M}\right)=\left(N, F_{N}\right)$, then we have $M=N$, where $M=\operatorname{supp}\left(M, F_{M}\right), N=\operatorname{supp}\left(N, F_{N}\right)$.

Proposition $1.2([3])$. If $\left(M, F_{M}\right) \subseteq\left(N, F_{N}\right)$, then we have $M \subseteq N$, where $M=\operatorname{supp}\left(M, F_{M}\right), N=\operatorname{supp}\left(N, F_{N}\right)$.

We also need the following notations and results from the classical complex analysis [5].

For $D \subset \mathbb{C}$, we denote by $\mathcal{H}(D)$ the class of holomorphic functions on $D$, and by $\mathcal{H}_{n}(D)$ the class of holomorphic and univalent functions on $\mathrm{D}$.

In this paper, we denote by $\mathcal{H}(U)$ the set of holomorphic functions in the unit disc $U=\{z \in \mathbb{C}:|z|<1\}$ with $\partial U=\{z \in \mathbb{C}:|z|=1\}$ the boundary of the unit disc.

For $a \in \mathbb{C}$ and $n \in \mathbb{N}$ we denote

2000 Mathematics Subject Classification. Primary: 30C80.

Key words and phrases. Fuzzy set, fuzzy subordination, fuzzy differential subordination, fuzzy best dominant. 


$$
\begin{gathered}
\mathcal{H}[a, n]=\left\{f \in \mathcal{H}(U): f(z)=a+a_{n} z^{n}+a_{n+1} z^{n+1}+\ldots, z \in U\right\}, \\
A_{n}=\left\{f \in \mathcal{H}(U): f(z)=z+a_{n+1} z^{n+1}+\ldots, z \in U\right\} \text { with } A_{1}=A, \\
\text { and } S=\{f \in A: f \text { a univalent function in } U\} .
\end{gathered}
$$

Let $\mathcal{B}=\{\varphi \in \mathcal{H}(U): \varphi(0)=0,|\varphi(z)|<1, z \in U\}$ denote the class of Schwarz functions.

Definition $1.2([4])$. Let $f, g \in \mathcal{H}(U)$. We say that the function $f$ is subordinated to $g$, written $f<g$ or $f(z)<g(z)$ if there exists a function $w \in \mathcal{H}(U)$ with $w(0)=0$ and $|w(z)|<1, z \in U$, (which means $w \in \mathcal{B}$ ) such that $f(z)=g(w(z)), z \in U$.

Let $D \subset \mathbb{C}$ and $f, g \in \mathcal{H}(D)$ holomorphic functions. We denote by $f(D)=\left\{f(z) \mid 0<F_{f(D)} f(z) \leq 1, z \in D\right\}=\operatorname{supp}\left(f(D), F_{f(D)}\right)$

and

$$
g(D)=\left\{g(z) \mid 0<F_{g(D)} g(z) \leq 1, z \in D\right\}=\operatorname{supp}\left(g(D), F_{g(D)}\right) .
$$

Definition 1.3 ([5]). Let $D \subseteq \mathbb{C}, z_{0} \in D$ be a fixed point, and let the functions $f, g \in \mathcal{H}(D)$. The function $f$ is said to be fuzzy subordinate to $g$ and write $f<{ }_{\boldsymbol{F}} g$ or $f(z)<_{\boldsymbol{F}} g(z)$, if

1. $f\left(z_{0}\right)=g\left(z_{0}\right)$,

2. $F_{f(D)} f(z) \leq F_{g(D)} g(z), z \in D$.

Proposition $1.3([5])$. Let Let $D \subset \mathbb{C}, z_{0} \in D$ be a fixed point, and let the functions $f, g \in \mathcal{H}(D)$. If $f(z)<_{\mathbf{F}} g(z), z \in D$, then

1. $f\left(z_{0}\right)=g\left(z_{0}\right)$

2. $f(D) \subseteq g(D)$, where $f(D)=\operatorname{supp}\left(f(D), F_{f(D)}\right), g(D)=\operatorname{supp}\left(g(D), F_{g(D)}\right)$. The equality occurs if and only if $F_{f(D)} f(z)=F_{g(D)} g(z)$. Denoted by

$$
S^{*}=\left\{f \in A: \operatorname{Re} \frac{z f^{\prime}(z)}{f(z)}>0, z \in U\right\}
$$

the class of normalized starlike functions in $U$,

$$
K=\left\{f \in A: \operatorname{Re} \frac{z f^{\prime \prime}(z)}{f^{\prime}(z)}+1>0, z \in U\right\}
$$

the class of normalized convex functions in $U$ and by

$$
C=\left\{f \in A: \exists \varphi \in K, \operatorname{Re} \frac{f^{\prime}(z)}{\varphi^{\prime}(z)}>0, z \in U\right\}
$$

the class of normalized close-to-convex functions in $U$ [5].

Let $J(\alpha, f ; z)=(1-\alpha) \frac{z f^{\prime}(z)}{f(z)}+\alpha\left(1+\frac{z f^{\prime \prime}(z)}{f^{\prime}(z)}\right), z \in U$, for $\alpha$ real number and $f \in A_{p}[2]$.

Let $\Omega=\operatorname{supp}\left(\Omega, F_{\Omega}\right)=\left\{z \in \mathbb{C}: 0<F_{\Omega}(z) \leq 1\right\}$,

$\Delta=\operatorname{supp}\left(\Delta, F_{\Delta}\right)=\left\{z \in \mathbb{C}: 0<F_{\Delta}(z) \leq 1\right\}, p(U)=\operatorname{supp}\left(p(U), F_{P(U)}\right)$

$\left.=\left\{f(z): 0<F_{P(U)}(f(z)) \leq 1\right\}, z \in U\right\}$ and

$\psi\left(\mathbb{C}^{3} \times U\right)=\operatorname{supp}\left(\psi\left(\mathbb{C}^{3} \times U\right), F_{\psi\left(\mathbb{C}^{3} \times U\right)}\right)$

$=\left\{\psi\left(p(z), z p^{\prime 2} p^{\prime \prime}(z) ; z\right): 0<F_{\psi\left(\mathbb{C}^{3} \times U\right)}\left(\psi\left(p(z), z p^{\prime 2} p^{\prime \prime}(z), z\right)\right) \leq 1, z \in U\right\}$ [4]. 
Definition $1.4([4])$. Let $\psi: \mathbb{C}^{3} \times U \rightarrow \mathbb{C}$ and let $h$ be univalent in $U$. If $p$ is analytic in $U$ and satisfies the (second-order) fuzzy differential subordination

$$
F_{\psi\left(\mathbb{C}^{3} \times U\right)}\left(\psi\left(p(z), z p^{\prime}(z), z^{2} p^{\prime \prime}(z) ; z\right) \leq F_{h(U)} h(z)\right.
$$

i.e. $\psi\left(p(z), z p^{\prime}(z), z^{2} p^{\prime \prime}(z) ; z\right)<_{\boldsymbol{F}} h(z), z \in U$, then $p$ is called a fuzzy solution of the fuzzy differential subordination. The univalent function $q$ is called a fuzzy dominant of the fuzzy solutions of the fuzzy differential subordination, or more simple a fuzzy dominant, if $p(z)<_{\boldsymbol{F}} g(z), z \in U$, for all $p$ satisfying (1). A fuzzy dominant $\tilde{q}$ that satisfies $\tilde{q}(z)<_{\boldsymbol{F}} q(z), z \in U$, for all fuzzy dominant $q$ of (1) is said to be the fuzzy best dominant of (1).

Theorem 1.1 ([5]). Let $h$ be analytic in $U$, let $\phi$ be analytic in domain $D$ containing $h(U)$ and suppose

a) $\operatorname{Re} \phi[h(z)]>0, z \in U$ and

b) $h(z)$ is convex.

If $p$ is analytic in $U$, with $p(0)=h(0), p(U) \subset D$ and $\psi\left(\mathbb{C}^{2} \times U\right) \rightarrow \mathbb{C}, \psi\left(p(z), z p^{\prime}(z)\right)=p(z)+z p^{\prime}(z) . \phi[p(z)]$ is analytic in $U$, then

implies

$$
F_{\psi\left(\mathbb{C}^{2} \times U\right)} \psi\left(p(z), z p^{\prime}(z)\right) \leq F_{h(U)} h(z),
$$

where

$$
F_{p(U)} p(z) \leq F_{h(U)} h(z), z \in U,
$$

$$
\begin{array}{r}
\psi\left(\mathbb{C}^{2} \times U\right)=\operatorname{supp}\left(\mathbb{C}^{2} \times U, F_{\psi\left(\mathbb{C}^{2} \times U\right)} \psi\left(p(z), z p^{\prime}(z)\right)\right. \\
=\left\{z \in \mathbb{C}: 0<F_{\psi\left(\mathbb{C}^{2} \times U\right)} \psi\left(p(z), z p^{\prime}(z)\right) \leq 1\right\}, \\
h(U)=\operatorname{supp}\left(U, F_{h(U)} h(z)\right)=\left\{z \in \mathbb{C}: 0<F_{h(U)} h(z) \leq 1\right\} .
\end{array}
$$

Theorem $1.2([5])$. Let $h$ be convex in $U$ and let $P: U \rightarrow \mathbb{C}$, with $\operatorname{Re} P(z)>$ 0 . If $p$ is analytic in $U$ and $\psi: \mathbb{C}^{2} \times U \rightarrow \mathbb{C}$,

$$
\psi\left(p(z), z p^{\prime}(z)\right)=p(z)+P(z) z p^{\prime}(z)
$$

is analytic in $U$, then

implies

$$
F_{\psi\left(\mathbb{C}^{2} \times U\right)}\left[p(z)+P(z) z p^{\prime}(z)\right] \leq F_{h(U)} h(z),
$$

$$
F_{p(U)} P(z) \leq F_{h(U)} h(z), z \in U .
$$

Theorem $1.3([5])$. (Hallenbeck and Ruscheweyh) Let $h$ be a convex function with $h(0)=a$, and let $\gamma \in \mathbb{C}^{*}$ be a complex number with Re $\geq 0$. If $p \in$ $\mathcal{H}[a, n]$ with $p(0)=a$ and $\psi: \mathbb{C}^{2} \times U \rightarrow \mathbb{C}, \psi\left(p(z)+z p^{\prime}(z)\right)=p(z)+\frac{1}{\gamma} z p^{\prime}(z)$ is analytic in $U$, then

$$
F_{\psi\left(\mathbb{C}^{2} \times U\right)}\left[p(z)+\frac{1}{\gamma} z p^{\prime}(z)\right] \leq F_{h(U)} h(z),
$$

implies

$$
F_{p(U)} p(z) \leq F_{q(U)} q(z) \leq F_{h(U)} h(z), z \in U
$$


where

$$
q(z)=\frac{\gamma}{n z^{\gamma / n}} \int_{0}^{z} h(t) t^{\frac{\gamma}{n}-1} \mathrm{~d} t
$$

The function $q$ is convex and is the fuzzy best $(a, n)$-dominant.

\section{Main Results}

Proposition 2.1. Let $q$ be univalent in $U$ and let $\theta$ and $\phi$ be analytic in a domain $D$ containing $q(U)$, with $\phi(w) \neq 0$, when $w \in q(U)$. Set $Q(z)=$ $z q^{\prime}(z) . \phi[q(z)]$ and $h(z)=\theta[q(z)]+Q(z)$ and suppose that either

(i) $Q$ is starlike, or

(ii) $h$ is convex.

In addition, assume that

(iii) $\operatorname{Re}\left(\frac{z h^{\prime}(z)}{Q(z}\right)=\operatorname{Re}\left(\frac{\theta^{\prime}[q(z)]}{\phi[q(z)]}+\frac{z Q^{\prime}(z)}{Q(z)}\right)>0$.

If $p$ is analytic in $U$, with $p(0)=q(0), p(U) \subset D$ and $\psi: \mathbb{C}^{2} \times U \rightarrow \mathbb{C}$, $\psi\left(p(z), z p^{\prime}(z)\right)=p(z)+z p^{\prime}(z) . \phi(p(z))$ is analytic in $U$, then

implies

$$
F_{\psi\left(\mathbb{C}^{2} \times U\right)}\left[p(z)+z p^{\prime}(z) \cdot \phi(p(z)] \leq F_{h(U)} h(z),\right.
$$

$$
F_{p(U)} p(z) \leq F_{q(U)} q(z), z \in U, \text { i.e. }
$$

$p(z)<_{\mathbf{F}} q(z)$, and $q$ is the best dominant, where

$\psi\left(\mathbb{C}^{2} \times U\right)=\operatorname{supp}\left(\mathbb{C}^{2} \times U, F_{\psi\left(\mathbb{C}^{2} \times U\right)} \psi\left(p(z), z p^{\prime}(z)\right)\right)$

$=\left\{z \in \mathbb{C}: 0<F_{\psi\left(\mathbb{C}^{2} \times U\right)} \psi\left(p(z), z p^{\prime}(z)\right) \leq 1\right\}$, and

$h(U)=\operatorname{supp}\left(U, F_{h(U)} h(z)\right)=\left\{z \in \mathbb{C}: 0<F_{h(U)} h(z) \leq 1\right\}$.

Proof. The proof of Proposition is similar to Theorem 1.1[5].

Proposition 2.2. Let $q \in \mathcal{H}[p, p]$ be univalent, $q(z) \neq 0$ and satisfies the following conditions.

(i) $\frac{z q^{\prime}(z)}{q(z)}$ is starlike,

(ii) $\operatorname{Re}\left(\frac{q(z)}{\alpha}+1+\frac{z q^{\prime \prime}(z)}{q^{\prime}(z)}-\frac{z q^{\prime}(z)}{q(z)}\right)>0$ for all $\alpha \neq 0$ and for all $z \in U$.

For $p \in \mathcal{H}[p, p]$ with $p(z) \neq 0$ in $U$ and

$$
\left.\psi: \mathbb{C}^{2} \times U \rightarrow \mathbb{C}, \psi p(z), z p^{\prime}(z)\right)=p(z)+\alpha \frac{z p^{\prime}(z)}{p(z)}
$$

is analytic in $U$, then

$$
F_{\psi\left(\mathbb{C}^{2} \times U\right)}\left[p(z)+\alpha \frac{z p^{\prime}(z)}{p(z)}\right] \leq F_{\psi\left(\mathbb{C}^{2} \times U\right)}\left[q(z)+\alpha \frac{z q^{\prime}(z)}{q(z)}\right]=F_{h(U)} h(z),
$$

implies

$$
F_{p(U)} p(z) \leq F_{q(U)} q(z) \text { i.e. } p(z)<_{\mathbf{F}} q(z), z \in U
$$

and $q$ is the best dominant.

Proof. Define the function $\theta$ and $\phi$ by $\theta(w)=w, \phi(w)=\frac{\alpha}{w}, D=\{w: w \neq 0\}$ in Proposition 2.1. Then the functions

$$
Q(z)=z q^{\prime}(z) \phi[q(z)]=\alpha \frac{z q^{\prime}(z)}{q(z)}
$$




$$
h(z)=\theta[q(z)]+Q(z)=q(z)+\lambda \frac{z q^{\prime}(z)}{q(z)} .
$$

Since $\frac{z q^{\prime}(z)}{q(z)}$ is starlike, we obtain that $Q$ is starlike in $U$ and $\operatorname{Re}\left(\frac{z h^{\prime}(z)}{Q(z)}\right)>0$ for all $z \in U$. It follows Proposition 2.1 and

$$
\begin{gathered}
F_{\psi\left(\mathbb{C}^{2} \times U\right)}\left[p(z)+\alpha \frac{z p^{\prime}(z)}{p(z)}\right] \leq F_{h(U)} h(z), \\
F_{p(U)} p(z) \leq F_{q(U)} q(z) \text { i.e. } p(z)<_{\boldsymbol{F}} q(z), z \in U,
\end{gathered}
$$

and $q$ is the best dominant.

Proposition 2.3. Let $q \in \mathcal{H}[p, p]$ be univalent, $q(z) \neq 0$ and satisties the conditions:

(i) $\frac{z q^{\prime}(z)}{q(z)}$ is starlike,

(ii) $\operatorname{Re}\left(\frac{q(z)}{\alpha}+1+\frac{z q^{\prime \prime}(z)}{q^{\prime}(z)}-\frac{z q^{\prime}(z)}{q(z)}\right)>0$

for $\alpha \neq 0$ and for all $z \in U$. For $f \in A_{p}$ with

$$
J(\alpha, f ; z)=(1-\alpha) \frac{z f^{\prime}(z)}{f(z)}+\alpha\left(1+\frac{z f^{\prime \prime}(z)}{f^{\prime}(z)}\right), z \in U
$$

and $\psi: \mathbb{C}^{2} \times U \rightarrow \mathbb{C}$,

$$
\begin{gathered}
\psi\left(q(z), z q^{\prime}(z)\right)=q(z)+\alpha \frac{z q^{\prime}(z)}{q(z)}, \text { then } \\
F_{\psi\left(\mathbb{C}^{2} \times U\right)}\left(\frac{z f^{\prime}(z)}{f(z)}\right) \leq F_{q(U)} q(z)
\end{gathered}
$$

and $q$ is the best dominant.

Proof. Let us put $p(z)=\frac{z f^{\prime}(z)}{f(z)}, z \in U$, where $\mathrm{p}(0)=0$.

Then we obtain that

$$
p(z)+\alpha \frac{z p^{\prime}(z)}{p(z)}=J(\alpha, f ; z) .
$$

Using Proposition 2.1, we have

$$
F_{p(U)} p(z) \leq F_{q(U)} q(z), z \in U,
$$

and $q$ is the best dominant.

Proposition 2.4. Let $q \in \mathcal{H}[1,1]$ be univalent and satisfies the following conditions:

(i) $q(z)$ is convex,

(ii) $\left.\operatorname{Re}\left[\left(\frac{1}{\alpha}+\rho\right)+\frac{z q^{\prime \prime}(z)}{q^{\prime}(z)}\right]>0 \rho \in \mathbb{N}=\{1,2,3, .\}.\right)$

for $\alpha \neq 0$ and for all $z \in U$. For $p \in \mathcal{H}[1,1]$ in $U$ and

$$
\begin{gathered}
\psi: \mathbb{C}^{2} \times U \rightarrow \mathbb{C}, \\
\begin{array}{c}
\psi\left(p(z), z p^{\prime}(z)\right)=(1-\alpha+\alpha \rho) p(z)+\alpha z p^{\prime}(z) \text { is analytic in } U, \text { then } \\
F_{\psi\left(\mathbb{C}^{2} \times U\right)}\left[(1-\alpha+\alpha \rho)\left(p(z)+\alpha z p^{\prime}(z)\right] \leq\right. \\
F_{\psi\left(\mathbb{C}^{2} \times U\right)}\left[(1-\alpha+\alpha \rho) q(z)+\alpha z q^{\prime}(z)\right]=F_{h(U)} h(z),
\end{array}
\end{gathered}
$$

implies $F_{p(U)} p(z) \leq F_{q(U)} q(z)$, and $q$ is the best dominant. 
Proof. For $\alpha \neq 0$ real number, we define the functions $\theta$ and $\phi$ by $\theta(w)=(1-\alpha+\alpha \rho) w, \phi(w)=\alpha, D=\{w: w \neq 0\}$ in Proposition 2.1.

Then we have

(i) $Q(z)=z q^{\prime}(z) \phi[q(z)]=\alpha z q^{\prime}(z)$,

(ii) $h(z)=\theta[q(z)+Q(z)]=(1-\alpha+\mu \rho) q(z)+\alpha z q^{\prime}(z)$.

By the (i)and (ii), we obtained that $Q$ is starlike in $U$ and $\operatorname{Re}\left(\frac{z h^{\prime}(z)}{Q(z)}\right)>0$ for all $z \in U$. Since it satisfies preconditions of Proposition 2.1, it follows Proposition 2.1,

$$
F_{p(U)} p(z) \leq F_{q(U)} q(z), z \in U,
$$

and $q$ is the best dominant.

Theorem 2.1. Let $q \in \mathcal{H}[1,1]$ be univalent and satisfies the following conditions:

(i) $q(z)$ is convex,

(ii) $\operatorname{Re}\left[\left(\frac{1}{\alpha}+\rho\right)+\frac{z q^{\prime \prime}(z)}{q^{\prime}(z)}\right]>0(\rho \in \mathbb{N}=\{1,2,3, .\}$.

for $\alpha \neq 0$ and for all $z \in U$. For $f \in A_{p}$ with

$$
J(\alpha, f ; z)=(1-\alpha) \frac{z f^{\prime}(z)}{f(z)}+\alpha\left(1+\frac{z f^{\prime \prime}(z)}{f^{\prime}(z)}\right), z \in U
$$

and if $\psi: \mathbb{C}^{2} \times U \rightarrow \mathbb{C}$,

$$
\begin{gathered}
\psi\left(q(z), z q^{\prime}(z)\right)=(1-\alpha+\alpha \rho) q(z)+\mu z q^{\prime}(z), \text { then } \\
F_{\psi\left(\mathbb{C}^{2} \times U\right)}\left(\frac{f(z)}{z^{p}}\right) \leq F_{q(U)} q(z), z \in U
\end{gathered}
$$

and $q$ is the best dominant.

Proof. Let us put $p(z)=\frac{f(z)}{z^{p}}$, where $p(0)=1$. Then we have

$$
(1-\alpha+\alpha \rho) p(z)+\alpha z p^{\prime}(z)=J_{p}(\alpha, f ; z) .
$$

From the Proposition 2.4, we have

$$
F_{p(U)} p(z) \leq F_{q(U)} q(z), z \in U
$$

and $q$ is the best dominant.

Corollary 2.1. Let $q \in \mathcal{H}[1,1]$ be univalent and satisfies the following conditions:

(i) $q(z)$ is convex,

(ii) $\operatorname{Re}\left[\left(\frac{1}{\alpha}+1\right)+\frac{z q^{\prime \prime}(z)}{q^{\prime}(z)}\right]>0(\rho \in \mathbb{N}=\{1,2,3, .\}$.

for $\alpha \neq 0$ and for all $z \in U$. For $p \in \mathcal{H}[1,1]$ in $U$,

if $\psi: \mathbb{C}^{2} \times U \rightarrow \mathbb{C}$

$\psi\left(p(z), z p^{\prime}(z)\right)=p(z)+\alpha z p^{\prime}(z)$,

then $F_{\psi\left(\mathbb{C}^{2} \times U\right)} p(z) \leq F_{q(U)} q(z), z \in U$, and $q$ is the best dominant. 
Corollary 2.2. Let $q \in \mathcal{H}[1,1]$ be univalent, $q(z)$ is convex for all $z \in U$. For $p \in \mathcal{H}[1,1]$ in $U$ if

$$
\begin{gathered}
\psi: \mathbb{C}^{2} \times U \rightarrow \mathbb{C}, \psi\left(p(z), z p^{\prime}(z)\right)=p(z)+z p^{\prime}(z), \text { then } \\
F_{\psi\left(\mathbb{C}^{2} \times U\right)} p(z) \leq F_{\psi\left(\mathbb{C}^{2} \times U\right)} q(z), z \in U,
\end{gathered}
$$

and $q$ is the best dominant.

Corollary 2.3. Let $q \in \mathcal{H}[1,1]$ be univalent, $q(z)$ is convex for all $z \in U$. For $p \in \mathcal{H}[1,1]$ in $U$ if

$$
\begin{gathered}
\psi: \mathbb{C}^{2} \times U \rightarrow \mathbb{C}, \psi\left(p(z), z p^{\prime}(z)\right)=\rho p(z)+z p^{\prime}(z),(\rho \in \mathbb{N}=\{1,2,3, . .\}), \\
\text { then } \\
F_{\psi\left(\mathbb{C}^{2} \times U\right)} p(z) \leq F_{\psi\left(\mathbb{C}^{2} \times U\right)} q(z), z \in U,
\end{gathered}
$$

and $q$ is the best dominant.

\section{REFERENCES}

[1] Ö.Ö. Kılıç, Sufficient Conditions for Subordination of Multivalent Functions, Journal of Inequalities and Applications, 2008 (2008), ArticleID 374756, 8 pages.

[2] S.S. Miller and P.T. Mocanu, Differential subordinations, Theory and applications, Marcel Dekker Inc., NewYork, Basel, 2000.

[3] G.I. Oros and Gh. Oros, The notion of subordination in fuzzy sets theory, General Mathematics, 19 (2011), 97-103.

[4] G.I. Oros and Gh. Oros, Fuzzy differential subordination, Acta Universitatis Apulensis, 30 (2012), 55-64.

[5] G.I. Oros and Gh. Oros, Dominants and best dominants in fuzzy differential subordinations, Stud. Univ. Babeş - Bolyai Math., 57 (2012), 239-248.

[6] L.A. Zadeh, Fuzzy sets, Information and Control, 8 (1965), 338-353.

\section{A. Haydar Eş}

Department of Mathematics Education

BAŞKENT UNIVERSITY

BAĞLICA

06490, ANKARA

TURKEY

E-mail address: haydares@baskent.edu.tr 